\title{
Experimental study of flow characteristics and mixing performance in a PZT self-pumping micromixer
}

\author{
H.J. Sheen*, C.J. Hsu, T.H. Wu, H.C. Chu, C.C. Chang, U. Lei \\ Institute of Applied Mechanics, National Taiwan University, No. 1, Section 4, Roosevelt Road, Taipei, Taiwan 106, ROC \\ Received 31 July 2006; received in revised form 22 December 2006; accepted 28 February 2007 \\ Available online 6 March 2007
}

\begin{abstract}
In this paper, a novel micromixer which possesses both pumping and mixing capabilities has been investigated by an externally triggered micro-PIV and the fluorescent tests. A mixing region with triangular-wave structures was integrated into an obstacle-type valveless micropump. The velocity and vorticity fields measured by micro-PIV indicated that the flow recirculation occurred upstream the obstacles and along the lateral direction of the triangular structures when the pump mode and supply mode were switched. Moreover, the distributions of recirculation regions varied rapidly due to the high frequency oscillating flow, which had significant effects in the mixing process. The mixing indices obtained from the mixture of fluorescent and blue dyes exhibited that the mixing region which is $2100 \mu \mathrm{m}$ in length provided the best mixing effects. The advantages such as rapid and uniform mixing, device portability, easy fabrication, etc. were all attainable in this self-pumping micromixer. In the future, the pumping and mixing performances can be optimized based on the quantitative measure technologies.
\end{abstract}

(c) 2007 Elsevier B.V. All rights reserved.

Keywords: PZT self-pumping micromixer; Externally triggered micro-PIV; Flow recirculation

\section{Introduction}

In recent years, many different types of micromixers have been developed for microfluidic-based biomedical and chemical analysis systems. Biological processes, for instance, polymerase chain reaction (PCR), DNA hybridization, cell activation, enzyme reactions, and protein folding all require a high efficient mixing for initiation. A micromixer is also an essential element in a versatile microfluidic chip such as micro-totalanalysis-system ( $\mu \mathrm{TAS})$. Therefore, a novel micromixer design requires some considerations such as rapid mixing, small size of devices, and good integration ability.

How to accomplish high mixing efficiency is the primary challenge of a micromixer design because the intermolecular diffusion dominates the mixing behaviors at low Reynolds number. Two categories of micromixers are mainly adopted to enhance mixing: passive and active types. The passive micromixers such as T-type, serpentine microchannel, modified Tesla structures, and embedded barrier [1-4] utilize the different geometries to

\footnotetext{
* Corresponding author. Tel.: +88623366 5632; fax: +88623366 5633 E-mail address: sheenh@ntu.edu.tw (H.J. Sheen).
}

maximize the interfacial surface area. However, high-pressure loss is expected for a complex structure design. On the other hand, the external forces or moving parts are needed to induce mixing in active micromixers. For instance, ultrasonic vibration, magneto hydrodynamic (MHD) stirrer, and ac electroosmotic flow [5-7] have been investigated to perturb the fluids. Moreover, a review on micromixers and mixing principles has been presented by Hessek et al. [8].

Previous study [9] has shown the feasibility of a selfpumping micromixer which is composed of a PZT (piezoelectric leas-zirconate-titanate) valveless micropump and passive microstructures. Both fluids transport and mixing effects were carried out at the same time. MEMS fabrication processes and simple patterns enable the self-pumping micromixers to be mass-produced in a cost-effective way. Another desirable trait of these micromixers is that they can be incorporated easily in portable biomedical analysis systems. In this study, an externally triggered micro-particle image velocimetry (micro-PIV) was used to study the flow characteristics. Furthermore, the emission of fluorescence was used to verify the performance in the mixing process. The quantitative results contributed to the study of mixing mechanism and can be used to improve effectiveness of pumping and mixing. 


\begin{tabular}{|ll|}
\hline \multicolumn{2}{|l|}{ Nomenclature } \\
\multicolumn{2}{|l|}{ Symbols } \\
$D$ & channel width $(\mu \mathrm{m})$ \\
$D_{\mathrm{f}}$ & diffusion coefficient $\left(\mathrm{mm}^{2} / \mathrm{s}\right)$ \\
$\bar{I}$ & mean intensity value \\
$I(j)$ & intensity at a cross-section \\
$L$ & characteristic length $(\mathrm{mm})$ \\
$M_{i}$ & mixing index \\
$N$ & number of total pixels \\
$P e$ & Peclet number \\
$R e$ & Reynolds number \\
$t$ & specific time phase in an operating cycle \\
$T$ & period of an operating cycle \\
$u$ & velocity in $x$ component $(\mathrm{mm} / \mathrm{s})$ \\
$v$ & velocity in $y$ component $(\mathrm{mm} / \mathrm{s})$ \\
$w_{z}$ & vorticity in $z$ component \\
\hline
\end{tabular}

\section{Experiments}

\subsection{Design and fabrication}

Fig. 1 illustrates the schematic diagram of the self-pumping micromixer. A PZT valveless micropump with asymmetric obstacles served as the driving source. A circular PZT disc of an $8 \mathrm{~mm}$ diameter was placed on the silicon plate of the mixer to deform the chamber. Two fixed-valves with opening angles of $7^{\circ}$ formed by the trapezoid obstacles and the channel wall were designed based on the nozzle-diffuser principle [10]. The throat between the obstacle and the side-wall was $40 \mu \mathrm{m}$. This element provided the direction-dependent flow resistances. Therefore, more flows were obtained in the diverging-direction, i.e., in the downstream direction than in the converging-direction. The width and depth of the microchannel were 500 and $80 \mu \mathrm{m}$, respectively.

On the microchip, there were two inlets for injected solutions and one outlet for the mixture. A mixing region was between the first obstacle and the chamber. In the mixing region, the $2 \mathrm{D}$ triangular-wave structures were staggered on the side-walls to enhance mixing. The triangular structure was $150 \mu \mathrm{m}$ in height.

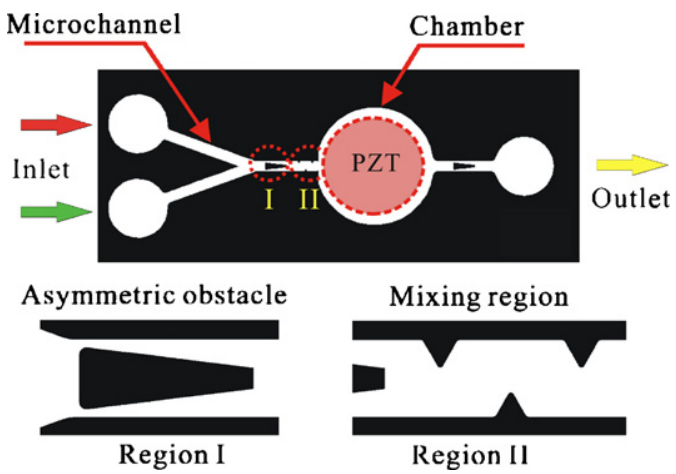

Fig. 1. The schematic diagram of the self-pumping micromixer including two asymmetrical obstacles and one mixing region with triangular-wave structures.

\section{(a) Clean wafer

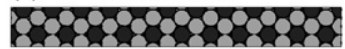

(b) AZP4620 spin

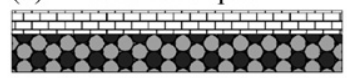

(c) Exposure (UV light)
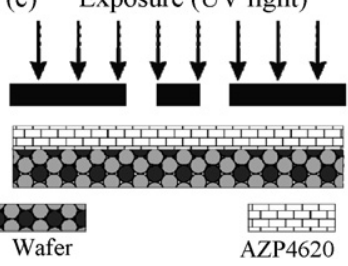

(d) Develop

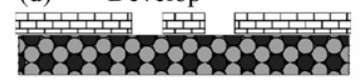

(e) Dry etching

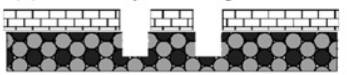

(f) Remove photoresist
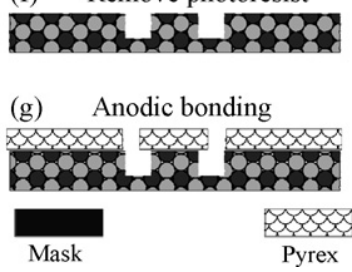

Fig. 2. The fabrication procedure of a self-pumping micromixer.

The distance between two adjacent triangular structures on the same side was $450 \mu \mathrm{m}$. The length of the mixing region is extremely short $(2100 \mu \mathrm{m})$, from the first triangular structure to the inlet of the pumping chamber. The $2 \mathrm{D}$ triangular-wave structures were selected here because those gave better bubble tolerance and caused less flow resistance than the square-wave ones.

The other important reason why the triangular-wave structures were adopted here is that the additional perturbation of reciprocated flow is expected. Vortices generated by the reciprocated motion of a triangular structure in the traditional flow have been presented by PIV measurement [11]. The vortices emanated and moved along the lateral of the triangular structure. A recent study also proposed a mixing mechanism by using the reciprocated flow to pass a sawtooth structure [12]. The mixing effects were thus enhanced even at a cycling rate of $1 \mathrm{~Hz}$. Therefore, the mixing efficiency can be drastically rose if these flow characteristics are applied in our micromixer.

The fabrication procedure of the micromixer is shown in Fig. 2. A single polished silicon wafer with a thickness of $500 \mu \mathrm{m}$ was used in the fabrication of the micromixer. The photoresist AZ-P4620 was spun on the wafer and only one photomask was required for the photolithography step. An inductive-couple-plasma (ICP) etcher which is suitable for high aspect ratio anisotropic etching was used after the patterns were exposed and developed. The microchannels were encapsulated by anodic bonding of a silicon wafer to the glass (Pyrex 7740) at a temperature of $400^{\circ} \mathrm{C}$. Finally, the PZT plate with a thickness of $200 \mu \mathrm{m}$ was adhered on the chamber. The holes drilled on the silicon wafer were sealed by glass tubes. Therefore, the pump pressure and volume flow rate can thus be evaluated.

\subsection{Velocity and vorticity fields by externally triggered micro-PIV}

The microflow characteristics were measured by an externally triggered micro-PIV system. An Nd-YAG laser double pulsing at $15 \mathrm{~Hz}$ was used to illuminate the flow field. The fluorescent particles (Duke Scientific ${ }^{\mathrm{TM}}$ ) with mean diameter $1 \mu \mathrm{m}$ served as the seeding particles. The excitation peak and emis- 
sion peak are at 542 and $612 \mathrm{~nm}$, respectively. A high sensitive CCD having $1600 \times 1200$ pixels resolution was mounted on an inverted Nikon ${ }^{\mathrm{TM}}$ microscope to capture the images. A $10 \times$ object lens was used to observe the flow behaviors and to collect the fluorescence emission.

The flow rate of the pump is dependent on the operating frequency of the PZT plate. In this experiment, the maximum flow rate occurred at $2.2 \mathrm{kHz}$ for various voltage inputs. Two operating modes (pump mode and supply mode) existed in an operating cycle when the PZT disc deformed the chamber. Although the micro-PIV is an efficient instrument to measure flow characteristic, the sampling rate, $15 \mathrm{~Hz}$, is still much lower than the cycling rate of the pump such that the oscillating motion of the fluids cannot be directly obtained. Therefore, an externally triggered signal which can keep specific phase delay with respect to the driving voltage is required in this experiment. An $\mathrm{HP}^{\mathrm{TM}}$ function generator provided both square wave and externally triggered signals to an amplifier and synchronizer, respectively. The 10 times amplified square-wave voltage by the power amplifier was used to drive the PZT disc. The synchronizer served as a timing and control module in the micro-PIV system.

Various phases were set to investigate the unsteady flow in one operating cycle. The time interval between consecutive phases was $1 / 16 T$. Thus the flow motions, no matter in pump mode or supply mode, were measured in detail. One hundred pairs of images (frames A and B with a time interval of $5 \mu \mathrm{s}$ ) were captured and averaged to reduce the background noises. Velocity vectors were computed from the image pairs by the cross-correlation algorithm with Nyquist condition.

The fluorescent particles were diluted with depleted ion water to act as the working fluids. Based on a first-order inertial response to a constant flow, the particle response time was around $10^{-7} \mathrm{~s}^{-1}$. The error due to Brownian motion in the experiment was less than $1 \%$. A testing micropump was mounted on an experiment holder to keep the focus plane and the chip parallel. The arrangement of the instruments and micromixer are given in Fig. 3.

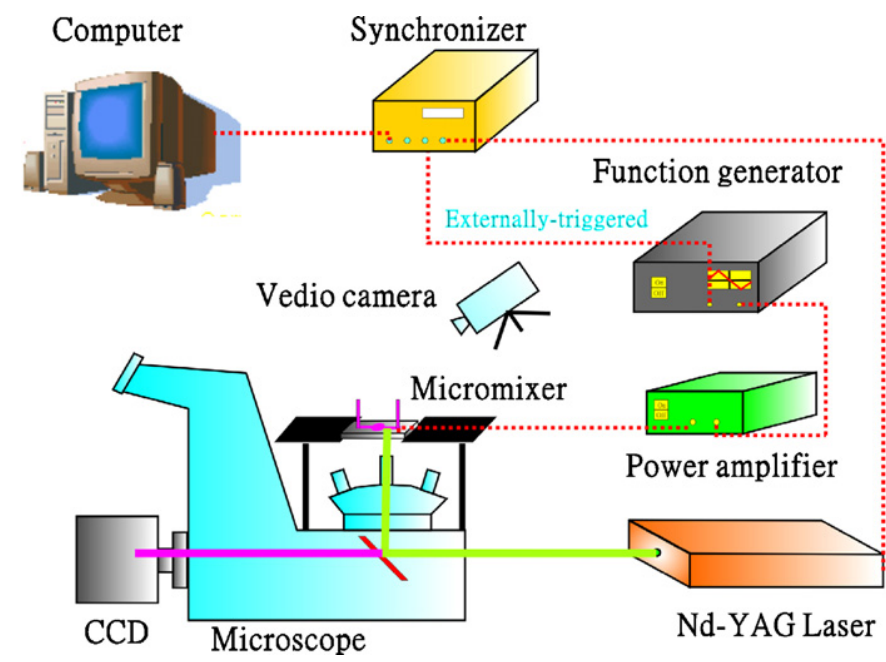

Fig. 3. The arrangement of the instruments and the self-pumping micromixer.

\subsection{Evaluation of mixing efficiency}

In order to observe the mixing behaviors, fluorescent and blue dyes were used in the experiment. The fluorescence intensity is not in direct proportion to the concentration. Therefore, the blue dye was chosen to mix with the fluorescence in order to absorb excess emission. The linear relation between the intensity and concentration was thus obtained. The images were recorded by a microscope and a CCD. The captured images were processed by digital image processing to count the pixels of each grey level.The estimation of the mixing efficiency is by analyzing the distribution of fluorescence intensities at a cross-section of a microchannel. The mixing index [13] which compares the grey level of each pixel along a cross-section and the mean value is given as follows:

$M_{I}=1-\frac{1}{\bar{I}} \sqrt{\frac{\sum(I(j)-\bar{I})^{2}}{N}}$

For uniform mixing, the deviation of the fluorescence intensity should be 0 , thus the mixing index being 1 . On the other hand, as the mixing index drops from 1 to 0 , less and less mixing accomplishes. A zero mixing index means no mixing. Therefore, the mixing indices are also useful to assess the effects of the mixing region.

\section{Results}

\subsection{Velocity and vorticity fields}

The unsteady flow field in the $x-y$ plane was successfully measured by the externally triggered micro-PIV system in both operating modes. In the supply mode, the flow sucked towards the chamber from both the inlet and outlet because of the underpressure in the expanded volume. The reverse result was obtained in the pump mode when the chamber was compressed. Two regions which had interesting phenomena were observed in the experiment: upstream region of the upstream obstacle (region I) and the triangular-wave mixing region (region II). The micro-PIV images of the two regions are shown in Fig. 4.

The velocity and vorticity field shown as follows were according to the operating condition of a $50 \mathrm{~V}$ input voltage. Similar results were also measured for lower voltage inputs. The fluids drawn from the two inlet glass tubes were in the same flow rate. Fig. 5(a)-(d) illustrates the velocity vectors in the region I at four different phases $0 T, 1 / 4 T, 1 / 2 T$, and 3/4T, respectively. The flow recirculation appeared upstream the first obstacle when the pump mode was changed to the supply mode as given in Fig. 5(a). The upper fluids rotated clockwise while the lower ones rotated counterclockwise. Moreover, the secondary recirculation regions with opposite rotations were between the primary ones and the channel walls. The flow recirculation was conducive to mixing because the fluid rotation increases the interfacial surface area by convection. The fluids in Fig. 5(b) flow rightward to the chamber in supply mode. The reversed flow in pump mode is obtained in Fig. 5(d). No recirculation flow was observed when the supply mode was changed to pump 

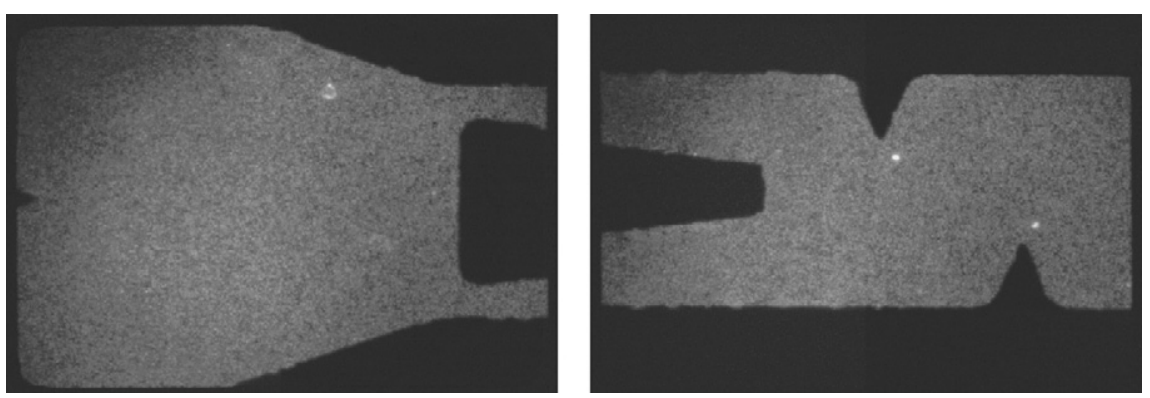

Fig. 4. The instantaneous images of two measure regions.

mode as shown in Fig. 5(c). The flow was smooth most of the time in a cycle.

The key in the design of this mixer is the triangular-wave structure with staggered arrangements in the mixing region. The velocity fields in Fig. 6(a)-(d) clearly demonstrate the mixing mechanism in the mixing region in a time period. The structures forced the fluids to move in the $y$-direction. Fig. 6(b) and (d) shows the supply and pump mode smooth flow field, respectively. The highest velocities appeared in the back region of the triangular structures when the fluids were accelerated towards the downstream direction. The high concentration materials were carried to the central axis from the side-wall. Furthermore, the recirculation flow patterns were also observed when the two operating modes were switched and shown in Fig. 6(a) and (c). The recirculation regions appeared at the upstream side of the triangular-wave structures when the pump mode was changed to the supply mode $(t=0 T)$. Very similar phenomenon was observed at the downstream side when the supply mode
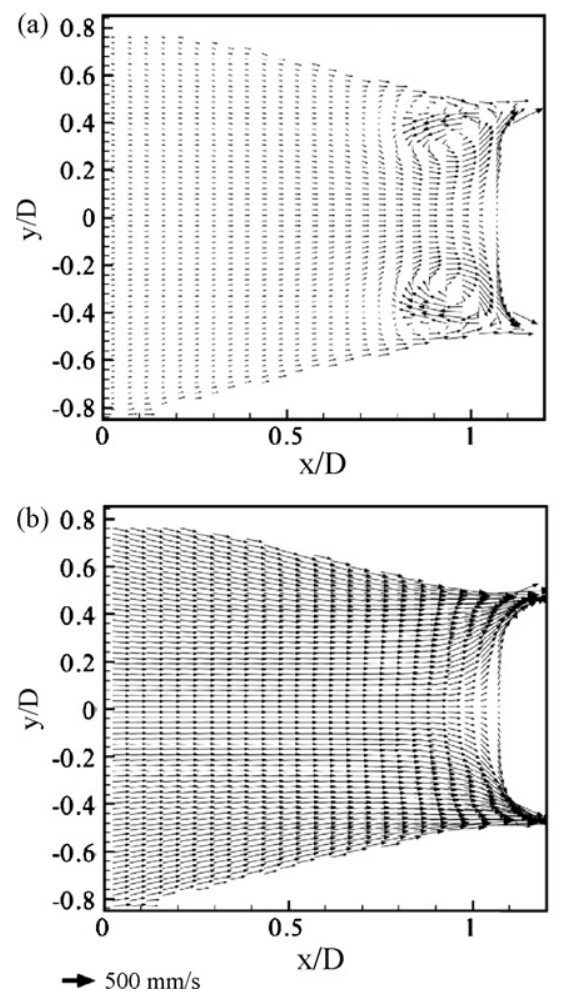

was switched to the pump mode $(t=1 / 2 T)$. The rotation flows effectively reduced the gradient of concentration in the radial direction and provided a nice mixing mechanism. The size of recirculation region was dependent on the lateral length of the triangular structure. Longer length can be thus used to induce larger size of recirculation region. Although this may enhance the mixing effects, the pump efficiency could be reduced due to additional flow resistance.

In order to study the mixing mechanism in detail, the distribution of vorticity is considered here. A vorticity is a vector with magnitude proportional to the strength of rotation. The $z$ component of a vorticity, $w_{z}$, is defined as

$w_{z}=\frac{\partial v}{\partial x}-\frac{\partial u}{\partial y}$

Positive values of $w_{z}$ correspond to counterclockwise rotation. The contours presented in Fig. 7(a)-(d) illustrate that the distributions of the vorticity upstream the first obstacle are in
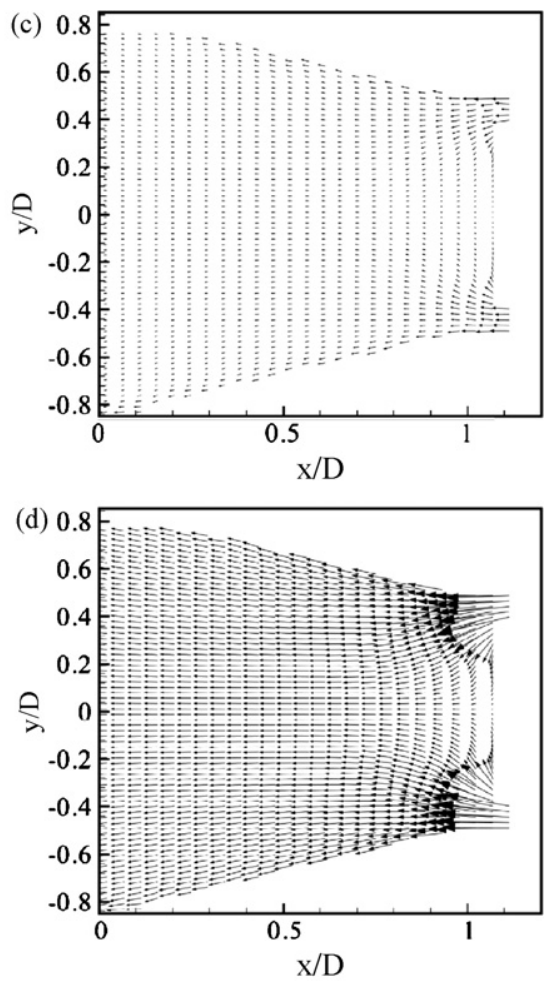

Fig. 5. The velocity vectors at four different phases in region I: (a) pump mode was changed to supply mode at $0 T$, (b) supply mode flow at $1 / 4 T$, (c) supply mode was changed to pump mode at $1 / 2 T$ and (d) pump mode flow at $3 / 4 T$. 

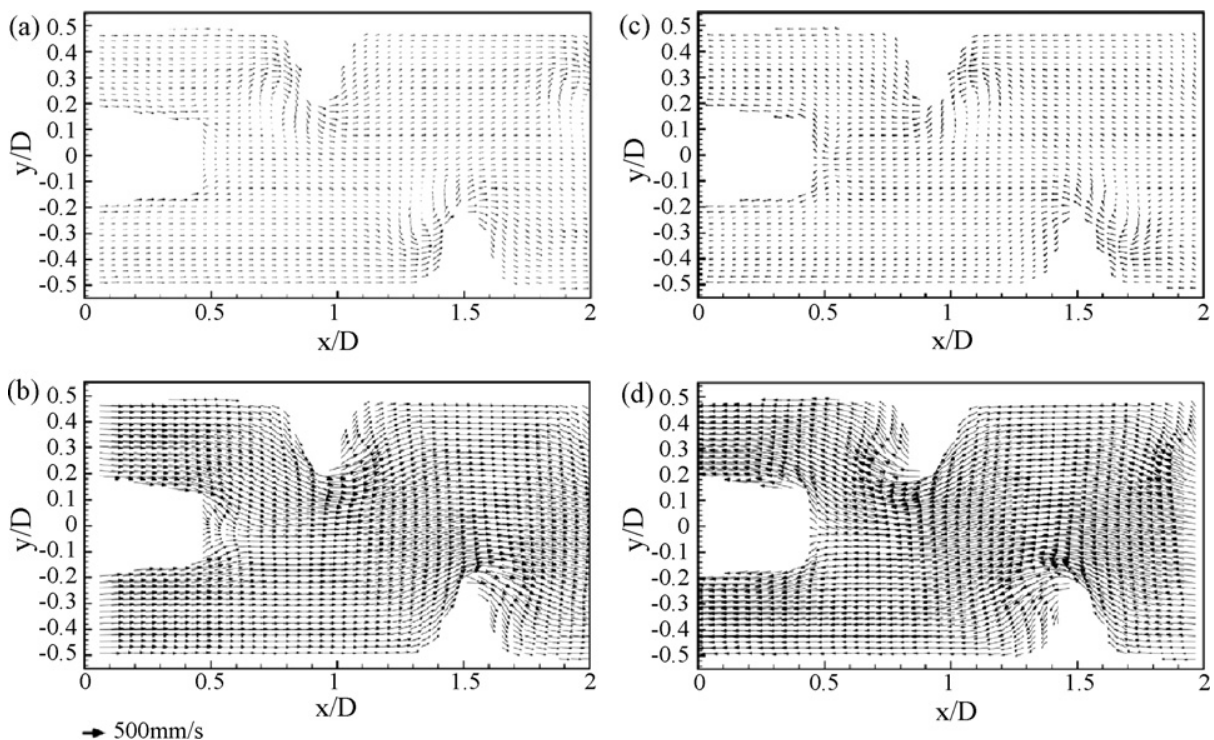

Fig. 6. The velocity vectors at four different phases in region II: (a) pump mode was changed to supply mode at $0 T$, (b) supply mode flow at $1 / 4 T$, (c) supply mode was changed to pump mode at $1 / 2 T$ and (d) pump mode flow at $3 / 4 T$.

accordance with the velocity field in Fig. 5(a)-(d). The red color and blue color designate the positive and negative vorticity, respectively. The high vorticity regions were generated upstream the throats at $0 T$ as shown in Fig. 7(a). The primary and secondary vorticity regions with opposite magnitude existed at both sides anti-symmetrically with respect to the central axis.
However, the magnitudes and sizes of the high vorticity regions were not noticeable most of the time upstream the obstacle as demonstrated in Fig. 7(b)-(d).

In the mixing region, high vorticity regions occurred in correspondence with the staggeringly arranged triangular-wave structures and their magnitudes were anti-symmetric with
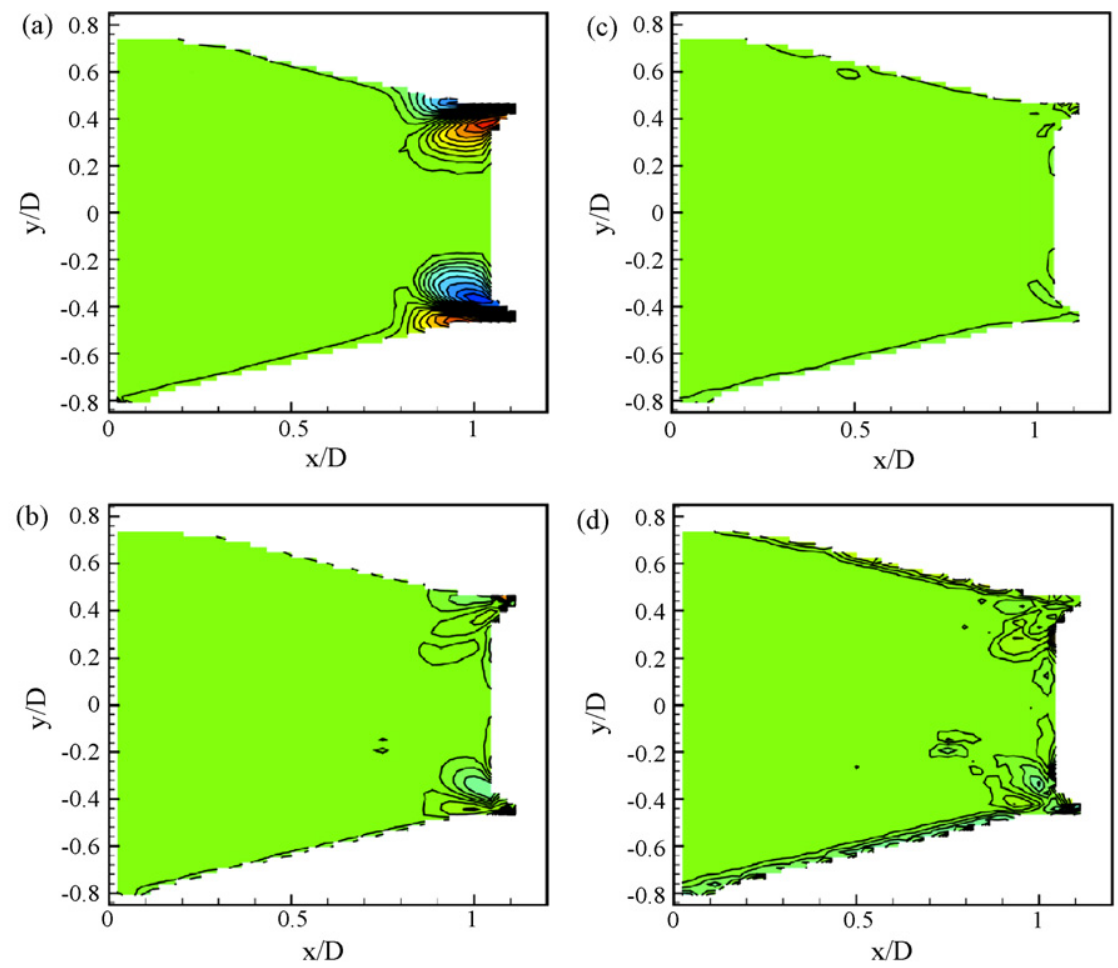

Vorticity: $-1.45 \mathrm{E}+04 \quad-9.92 \mathrm{E}+03 \quad-5.34 \mathrm{E}+03 \quad-7.63 \mathrm{E}+02 \quad-3.82 \mathrm{E}+03 \quad 8.39 \mathrm{E}+03 \quad 1.30 \mathrm{E}+04$

Fig. 7. The vorticity contours at four different phases in region I: (a) pump mode was changed to supply mode at $0 T$, (b) supply mode flow at $1 / 4 T$, (c) supply mode was changed to pump mode at $1 / 2 T$ and (d) pump mode flow at $3 / 4 T$. 

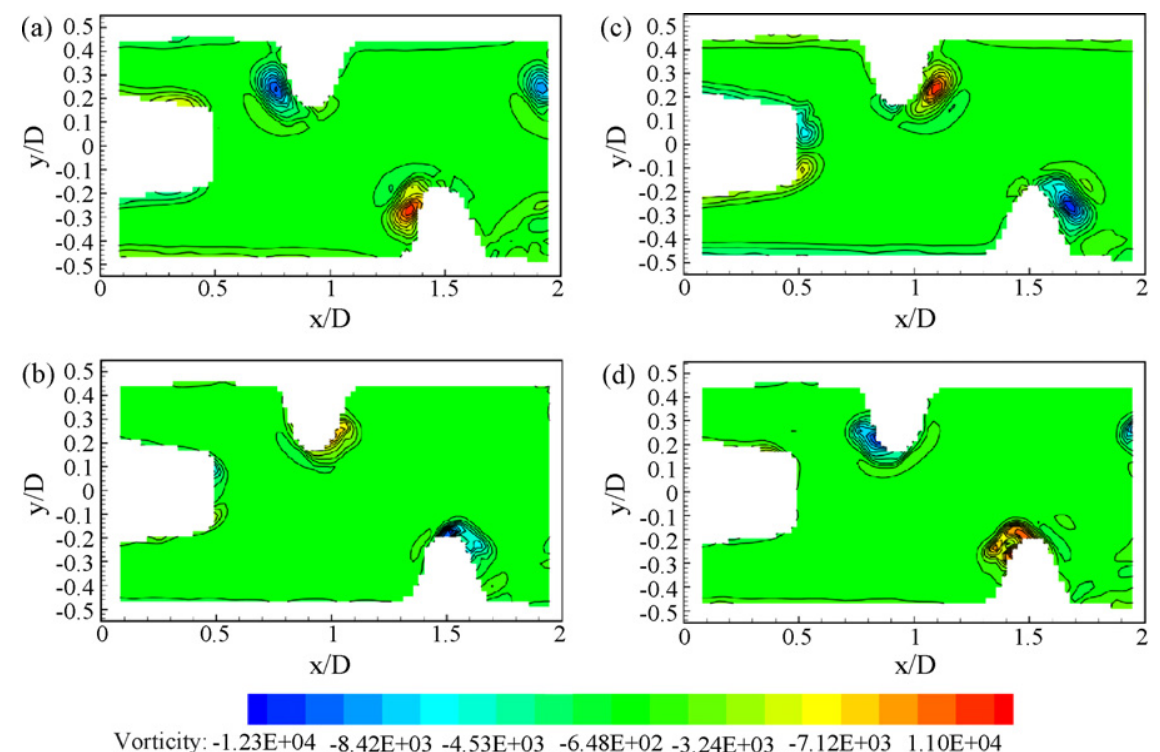

Fig. 8. The vorticity contours at four different phases in region II: (a) pump mode was changed to supply mode at $0 T$, (b) supply mode flow at $1 / 4 T$, (c) supply mode was changed to pump mode at $1 / 2 T$ and (d) pump mode flow at $3 / 4 T$.

respect to the central axis as shown in Fig. 8(a)-(d). In our test results, the spatial distributions of high vorticity regions were time-dependent.

Moreover, the high vortice regions in Fig. 8(a) and (c) were induced due to the flow rotations, while the others in Fig. 8(b) and (d) were induced due to the dramatic velocity gradient. The regular motion of the vorticity region was only observed in periodically unsteady flow in the microchannel. The high vorticity regions were important to the mixing efficiencies. The mixing mechanism due to the intermolecular diffusion was replaced by the high angular momentum fluids motion. The mixing efficiency was verified by two different dyes and will be discussed in the following section.

\subsection{Pumping and mixing performance}

Mixing and pumping were actualized at the same time when the square waves were put in to drive the PZT disc. The pump pressures were all higher than $800 \mathrm{~Pa}$ for all input voltages of 30-50 V. Fig. 9 shows that the flow rate obtained at the outlet increased when voltage inputs were raised linearly. The maximum flow rate obtained in the tests approached $50 \mu \mathrm{l} / \mathrm{min}$. The net volume flow depended on the difference of the forward and the backward flow rates per cycle. The mixing quality can be estimated by Peclet number, $P e$, which is determined by the ratio of the diffusive time to the residence time:

$P e=\frac{u L}{D_{\mathrm{f}}}$

where $L$ is the hydraulic diameter of the channel, and $D_{\mathrm{f}}$ is the mass diffusion constant here is $10^{-10} \mathrm{~m}^{2} / \mathrm{s}$. Table 1 gives the residence times and Peclet numbers in accordance with the test results of Fig. 9. A high Peclet number indicate that poor mixing is inevitable unless a passive or active device is used. In this experiment the flow recirculation resulted in a rapid and uniform mixing. This phenomenon will be discussed later.

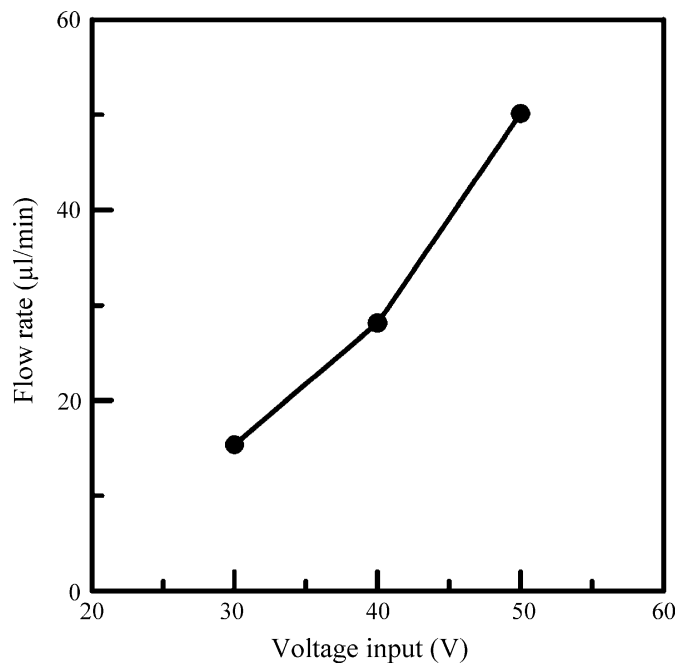

Fig. 9. The flow rates with various driving voltage inputs at $2.2 \mathrm{kHz}$.

The flow visualization by the fluorescent and blue dyes was utilized to examine the uniformity in various sections as shown in Fig. 10. The left photos reveal that the interfaces were clearly observed when the fluorescent and blue dyes began to mix at the confluence. A recirculation region occurred upstream the obstacle and its size enlarged as the excitation voltages were increased. However, the measured velocities in Fig. 5 indicated that the duration of the recirculation region, about $1 / 4$ cycle, was

\section{Table 1}

The residence times and Peclet numbers were evaluated based on the net flow velocity with various driving voltages

\begin{tabular}{llll}
\hline $\begin{array}{l}\text { Driving voltage } \\
\text { (V, peak to peak) }\end{array}$ & 30 & 40 & 50 \\
\hline Residence time (s) & 0.33 & 0.18 & 0.1 \\
$P e$ & $5.8 \times 10^{3}$ & $1.1 \times 10^{4}$ & $1.9 \times 10^{4}$ \\
\hline
\end{tabular}



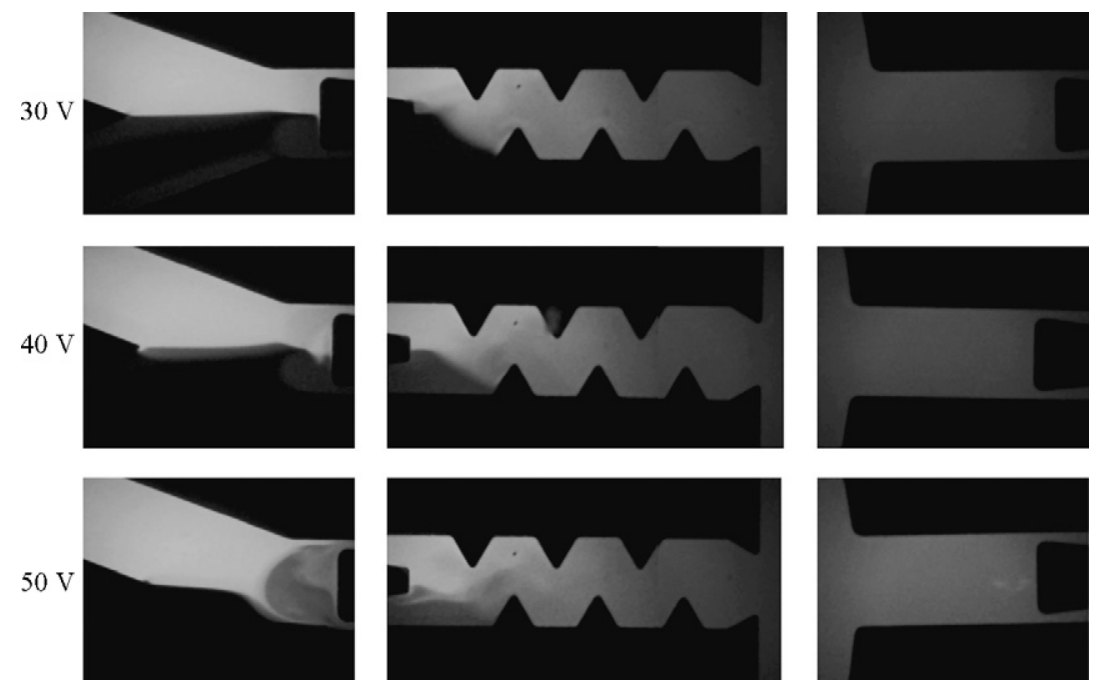

Fig. 10. Flow visualization by the fluorescent and blue dyes in various sections with three input voltages 30,40 and $50 \mathrm{~V}$.

too short to enhance the mixing effectively. In addition, due to the symmetric geometry of the inlet channels, most of the flow mixing occurred at the interface of the two fluids. However, the mixture was still in poor mixing when the fluid flow through the diffuser section. The images in the center of Fig. 10 show good mixing quality in the mixing region. Multiple recirculation zones behind the staggered triangular structures can be observed and their positions switched up and down in high frequency. The recirculation zones stirred the flow streams so that the diffusion effect was enhanced due to the increased interfacial area. The right photos exhibit that the fluorescent emission became uniform when the mixture flew through the second obstacle to the outlet.

In order to quantify the mixing efficiency, the mixing indices were evaluated along the streamwise direction as depicted in Fig. 11. The variations of the mixing indices in the three regions: upstream the mixing region (region $\mathrm{A}$ ), in the mixing region (region $\mathrm{B}$ ), and downstream the mixing region (region $\mathrm{C}$ ), clearly exhibit the mixing status. The mixing indices in region $\mathrm{A}$ increased from the inlet to the leading edge of the obstacle due to the flow recirculation. For the mixing region with staggered triangular structures, region $\mathrm{B}$, the mixing indices increased dramatically from 0.2 to 0.8 within a few milliseconds. However, the mixing indices became lower as the driving voltage was increased to result in a higher pumping flow rate. For region $\mathrm{C}$, the mixing effect was only resulted from the mass diffusion. The diffusion rates were decreased due to the smooth concentration gradients so that the mixing indices did not increase apparently in this region.

When the images were captured by the CCD, the camera dark noise and the readout noise led to a decrease of the mixing index. The former noise depends on the illumination condition and the operating temperature. The latter is an electronic noise as the output signals of CCD are converted into digital forms. A lower signal-to-noise ratio is expected for a darker light source, e.g. $50 \%$ fluorescence, i.e. the flow is in uniform mixing. Therefore, the maximum mixing index obtained in the experiment was 0.84 which was lower than the ideal value.

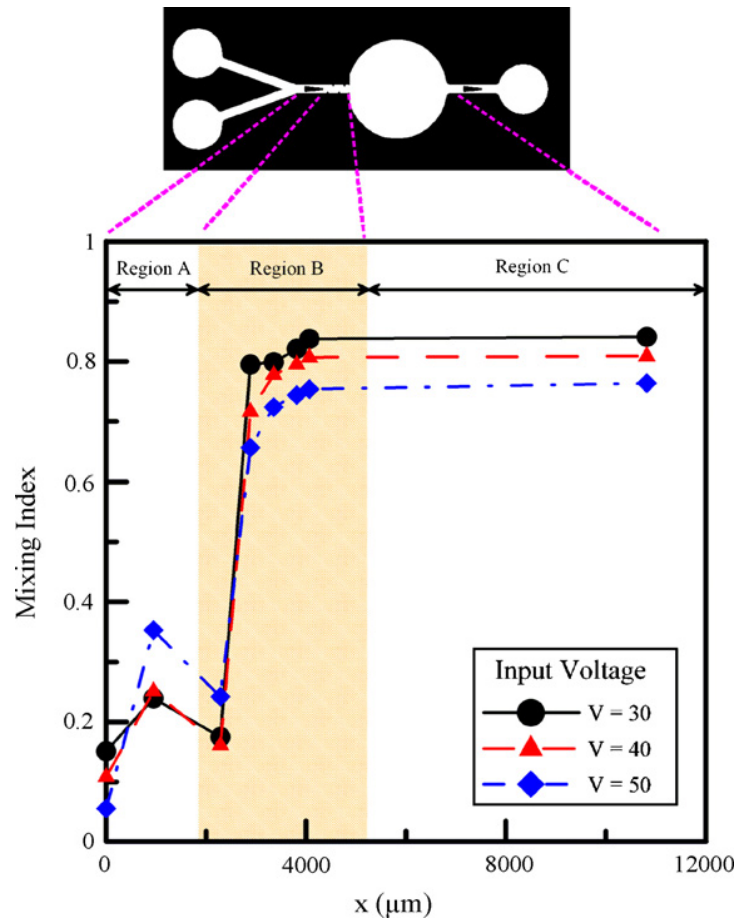

Fig. 11. The distribution of mixing indices in the micromixer with various input voltages. Region A: from the confluence of the two distinct fluids to the inlet of mixing region. Region $\mathrm{B}$ : the mixing region. Region $\mathrm{C}$ : from the inlet of pumping chamber to the upstream position of the second obstacle.

\section{Conclusions}

The characteristics of flow fields and mixing behaviors were explored by the externally triggered micro-PIV system and the tests of fluorescent-blue mixtures. Some important results are summarized as follows.

1. The velocity and vorticity fields indicated that flow is quite complex especially when the two operating modes are switched. Flow recirculation occurs when an oscillating flow 
passes a cusp or more generally an obstacle. The size and duration of the recirculation region depends on the operating conditions such as input voltages, frequencies, and the geometries. Although this kind of behavior provides the mixing mechanism in the mixer, the loss of pump efficiency must be estimated in the design work.

2. Both asymmetric obstacles and mixing region have contribution to mixing effects. The high frequency regular variation of high vorticity distributions enables the effective mixing to be achieved within a very short length in a very short time. The mixing indices also demonstrated that the high mixing performance can be obtained in the mixing region.

3. The quantitative measurements of both the flow fields and mixing efficiencies made substantial contribution to the study of mixing mechanisms. The geometries of the triangular-wave structures can be modified when we grasp the connection between the mixing effects and flow field. In the future, the flow recirculation regions will be anticipated at the suitable position such as the interface of the distinct fluids to enhance the mixing effectively.

\section{Acknowledgements}

This work is supported by Ministry of Economic Affairs, 94-EC-17-A-05-A1-0017, and National Science Council, NSC94-2120-M-002-014, of Taiwan, ROC.

\section{References}

[1] S.H. Wong, M.C.L. Ward, C.W. Wharton, Micro T-mixer as a rapid mixing micromixer, Sens. Actuators B: Chem. 100 (2004) 359-379.

[2] Y.Z. Liu, B.J. Kim, H.J. Sung, Two-fluid mixing in a microchannel, Int. J. Heat Fluid Flow 25 (2004) 986-995.

[3] C.C. Hong, J.W. Choi, C.H. Ahn, A novel in-plane passive microfluidic mixer with modified Tesla structure, Lab Chip 4 (2004) 109-113.

[4] D.S. Kim, S.W. Lee, T.H. Kwon, S.S. Lee, A barrier embedded chaotic micromixer, J. Micromech. Microeng. 14 (2004) 798-805.

[5] Z. Yang, S. Matsumoto, H. Goto, M. Matsumoto, R. Maeda, Ultrasonic micromixer for microfluidic systems, Sens. Actuators A: Phys. 93 (2001) 266-272.

[6] H.H. Bau, J. Zhong, M. Yi, A minute magneto dynamic (MHD) mixer, Sens. Actuators B: Chem. 79 (2001) 207-215.

[7] N. Sasaki, T. Kitamori, H.B. Kim, AC electroosmotic micromixer for chemical processing in a microchannel, Lab Chip 6 (2006) 550-554.
[8] V. Hessek, H. Löwe, F. Schönfeld, Micromixers-a review on passive and active mixing principles, Chem. Eng. Sci. 60 (2005) 2479 2501.

[9] C.J. Hsu, H.J. Sheen, T.H. Wu, H.C. Chu, C.C. Chang, U. Lei, Characteristics of Flow Field in a PZT Self-pumping Micromixer, in: Asia Pacific Conference of Transducers and Micro-Nano Technology, Singapore, 2006.

[10] E. Stemme, G. Stemme, A valve-less diffuser/nozzle based fluid pump, Sens. Actuators A: Phys. 39 (1993) 159-167.

[11] T.S. Jespersen, J.Q. Thomassen, A. Andersen, T. Bohr, Vortex dynamics around a solid ripple in an oscillatory flow, Eur. Phys. J. B 38 (2004) $127-138$.

[12] K.P. Nichols, J.R. Ferullo, A.J. Baeumner, Recirculating, passive micromixer with a novel sawtooth structure, Lab Chip 6 (2006) 242 246.

[13] Y.K. Lee, P. Tabeling, C. Shih, C.M. Ho, Characterization of a MEMSfabricated mixing device, in: ASME International Mechanical Engineering Congress \& Exposition, Orlando, FL, 2000, pp. 505-511.

\section{Biographies}

Dr. Horn-Jiunn Sheen is currently a professor in the Institute of Applied Mechanics, National Taiwan University. He is currently the director of the Nano-Electro-Mechanical-System (NEMS) Research Center, National Taiwan University. His research interests include micro-fluidics, bio-sensors, and flow measurements. The main research equipments and facilities in his laboratory are flow visualization systems, laser-Doppler velocimetry, micro-particle image velocimetry and a clean room (Class $10 \mathrm{k})$.

Chia-Jui Hsu was born in Taiwan in 1979. He received the BS degree and the MS degree in Institute of Aeronautics and Astronautics from National Cheng Kung University in 2001 and 2003, respectively. He is currently pursuing the $\mathrm{PhD}$ degree in Institute of Applied Mechanics, National Taiwan University. His research interests include microfluidics system and micro-PIV technique.

Tzu-Heng Wu received his BS degree in Mechanical Engineering from National Taiwan Ocean University in 2003 and MS degree in Institute of Applied Mechanics from National Taiwan University in 2005 . He is presently a $\mathrm{PhD}$ student in Institute of Applied Mechanics, National Taiwan University.

Hsin-Chang Chu received his BS degree in Mechanical Engineering from National Chung Cheng University in 2004 and MS degree in Institute of Applied Mechanics from National Taiwan University in 2006.

Che-Chun Chang received his BS degree and the MS degree in Department of System and Naval Mechatronic Engineering from National Cheng Kung University in 2002 and 2005, respectively.

Dr. U. Lei is currently a professor in the Institute of Applied Mechanics, National Taiwan University. His research interests include two-phase flow, micro-scale fluid mechanics, and nano-tribology. 